\title{
Beam shaping using Gaussian beam modes
}

\author{
John Lavelle* and Créidhe O'Sullivan \\ Department of Experimental Physics, National University of Ireland Maynooth, Co. Kildare, Ireland \\ *Corresponding author: john@quasioptics.com
}

Received October 29, 2009; accepted December 1, 2009;

posted December 17, 2009 (Doc. ID 118881); published January 29, 2010

\begin{abstract}
A beam shaping method is presented where a diffractive optical element (DOE) is designed by optimizing the complex mode coefficient weights of a set of Gaussian beam modes. This method is compared with the more standard unidirectional approach. Differential evolution is used for the optimization in both the unidirectional and Gaussian beam mode optimization methods. For the particular transforms carried out, the Gaussian beam mode set optimization (GBMSO) approach achieved more optimal solutions. The GBMSO approach is extended to design DOEs that control the amplitude distribution of a beam at multiple planes, rather than at just a single plane (i.e., the far field). (C) 2010 Optical Society of America
\end{abstract}

OCIS codes: $050.1970,260.1960$.

\section{INTRODUCTION}

Diffractive optical elements (DOEs) can be used to transform a coherent beam of light to some desired intensity pattern at another plane (for example, the far field) by imposing a phase distribution on a field [1]. The phase modulation of the DOE may be discrete or continuous. Discrete modulation may be necessitated by the manufacturing process, but may result in a less optimal solution (in terms of the far-field distribution achieved) compared to continuous profile. In this paper we consider continuous phase-only transmissive DOEs that impose a phase on the field by means of a profiled dielectric.

Due to the ill-posed nature of the problem, no analytical solution exists for finding the phase profile required to transform a given arbitrary input field to a given arbitrary output field, and in fact an exact transform may not even be possible [2]. Stochastic optimization algorithms, such as simulated annealing or genetic algorithms, have been applied to find optimal phase solutions [3,4]. The same algorithms that are applied to phase retrieval are used in DOE design. These algorithms can find solutions with the specified phase or amplitude constraints; however (depending on the target amplitude distribution), they can be computationally expensive [1].

First, this paper describes a typical method of DOE design where the phase of a scalar electric field, which was encoded as an array of elements, was optimized by iteratively modifying the phase, transforming to the far-field, and evaluating some merit function. Simulated annealing and differential evolution (DE) were both used to perform this optimization. Next, a method based on the optimization of a set Gaussian beam modes (GBMs) is introduced and the results are compared.

\section{DIFFERENTIAL EVOLUTION}

DE [5,6], a type of evolutionary strategy (ES), uses mechanisms inspired by biological evolution, whereby with each generation (or iteration), the "selection" of the "fitter individuals" from a "population" of designs is performed; perturbations (or "mutations") are then applied to the individuals, which are then "bred" by means of "crossover." Whereas genetic algorithms perform logical operations on bit strings, ESs perform arithmetic operations on floating point numbers. This makes them very suitable for a continuous parameter space optimization, as they are less complicated algorithms and provide greater control over the distribution of the mutant vector [5]. DE was used here for beam shaping, as it tends to be more robust than the more standard simulated annealing technique [7].

Only a brief description of $\mathrm{DE}$ is given here; a detailed description can be found in [5]. DE is generally initialized with a population of individuals, $x_{i, g}$, of index $i$ and generation $g$, each of which consists of parameters that encode the phase of the DOE. DE selects an individual labeling it $x_{r 0, g}$ (the base vector) and randomly selects two other individuals, which are labeled $x_{r 1, g}$ and $x_{r 2, g}$. A mutant vector is created as follows:

$$
v_{i, g}=x_{r 0, g}+F\left(x_{r 1, g}-x_{r 2, g}\right)
$$

where $F \in(0,1)$ is a real constant scaling factor, with a range recommended in [5].

DE then performs a type of discrete recombination, also known as the linear crossover, on each mutant vector of the intermediate population as follows:

$$
u_{i, g}= \begin{cases}v_{i, g} & \text { if }(\operatorname{rand}(0,1) \leq C)_{r} \\ x_{i, g} & \text { otherwise }\end{cases}
$$

where the crossover probability, $C_{r} \in[0,1]$, controls the fraction of parameters that are passed from each of the two randomly selected vectors to the trial vector. The optimal choice for parameters is problem specific; here a population size of $50, C_{r}=0.5$, and $F=0.5$ are used.

The objective function values of $v_{i, g}$ and $u_{i, g}$ are compared and the more optimal replaces $x_{r 0, g}$ in the next generation. All vectors (individuals) in turn are labeled $x_{r 0, g}$ and mutation, crossover, and selection are performed as 
above, before proceeding to the next generation. Mutation, crossover, and selection are performed on subsequent generations in this way until the convergence criterion-for example, that no improvement on the best solution is made for a given number of iterations-is met.

\section{UNIDIRECTIONAL OPTIMIZATION}

A Gaussian to rect( ) function far-field amplitude distribution transform was used here to compare the unidirectional and Gaussian beam mode set optimization (GBMSO) beam shaping methods. This transform is commonly used in the literature and is a challenging problem; beam shaping algorithms, such as the Gerchberg-Saxton algorithm or the unidirectional method, tend to get stuck at suboptimal solutions for this particular target function. Here, a $100 \mathrm{~mm}$ radius Gaussian beam with a flat phasefront was taken as the input field, and the DOE was used to obtain both $1^{\circ}$ and $10^{\circ}$ radii rect( ) function amplitude distributions in the far field.

In unidirectional optimization (also called the direct method), the field is transformed in one direction only (e.g., from the DOE plane to the far field) - as apposed to bidirectional optimization, such as the Gerchberg-Saxton algorithm [8], in which the field is also transformed in the conjugate direction. The DOE imposes a phase $\phi_{\mathrm{DOE}}(x, y)$ on the input field which has an amplitude distribution of $T_{i n}(x, y)$. In the Fraunhofer approximation, the far field is given by the Fourier transform as follows:

$$
E\left(k_{x}, k_{y}\right)=\mathcal{F}\left\{T_{\text {in }}(x, y) \exp \left[i \phi_{\mathrm{DOE}}(x, y)\right]\right\},
$$

where the Fourier transform operation $\mathcal{F}\{\}$ is computed using a fast Fourier transform (FFT) and $k$ 's are the spatial frequencies.

For simplicity, the optimization was restricted to the one-dimensional case. The DOE was divided into a number of discrete elements, $\phi_{\mathrm{DOE}}\left(x_{1}, 0\right), \phi_{\mathrm{DOE}}\left(x_{2}, 0\right), \ldots$, $\phi_{\mathrm{DOE}}\left(x_{N}, 0\right)$, as shown in Fig. 1 ; the depth of each of these elements was optimized using DE. The phase element, of depth $d$, imposes a phase $\phi_{\mathrm{DOE}}=2 \pi d(n-1) / \lambda$ on the input field, where $n$ is the refractive index of the DOE material. As a symmetric DOE was sought, each parameter in the optimization controlled the depth of two elements that were equidistant from the axis of symmetry (except the parameter that controls the central element). The radius of the DOE was $250 \mathrm{~mm}$ (2.5 times the radius of the Gaussian field incident on the DOE); the field outside of this region was padded with zeros.

A population of DOE designs, $\phi_{\mathrm{DOE}}\left(x_{i}, 0\right)$, imposing random phase values between 0 and $2 \pi$ was optimized. The

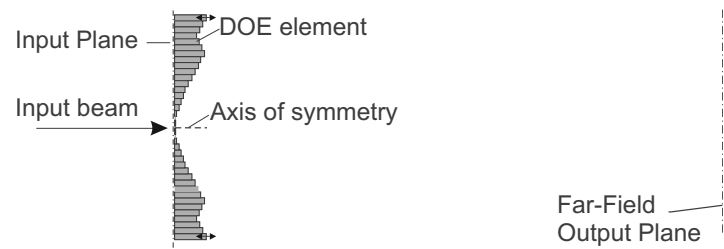

Fig. 1. For a DOE designed using unidirectional optimization, the global optimization algorithm optimizes the depth of each element of the DOE. following mean squared error (MSE) metric was used to quantify the design's "fitness:"

$$
\operatorname{MSE}=\frac{1}{N} \sum_{i=1}^{N}\left(E\left(k_{i}, 0\right)-T_{\text {out }}\left(k_{i}, 0\right)\right)^{2},
$$

where $E\left(k_{i}, 0\right)$ is the modeled far-field distribution, $T_{\text {out }}\left(k_{i}, 0\right)$ is the target far-field amplitude distribution, and $N$ is the number of samples in the field. Although it is the MSE that was minimized in the optimization, the power coupling (PC) between the optimized and target field distributions, assuming that the distributions have identical phases, is also quoted as it can be physically more meaningful. The $\mathrm{PC}$ is given by

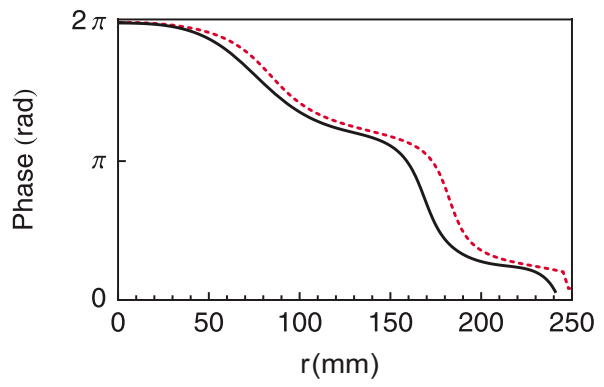

(a)

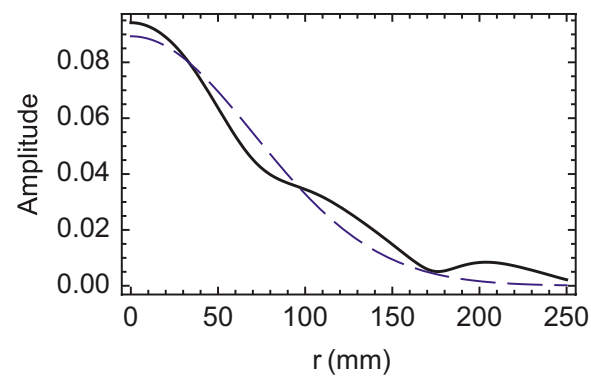

(b)

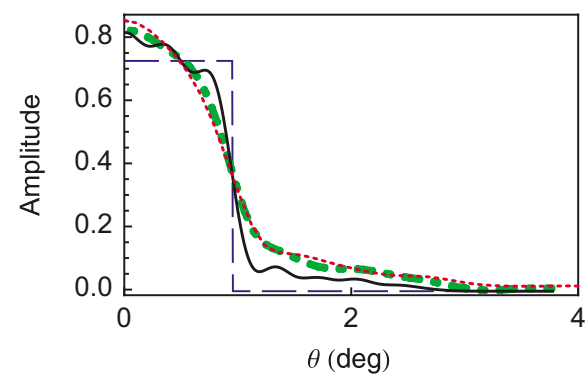

(c)

Fig. 2. (Color online) The results of the optimizations to transform a $100 \mathrm{~mm}$ radius Gaussian input field to a $1^{\circ}$ radius rect( ) function far-field distribution. (a) The phase that gives the DOE profile designed by unidirectional optimization (dotted line) and GBMSO (solid line). (b) The amplitude distribution given by the GBMs at the DOE plane (solid line) and the target amplitude distribution (dashed line). (c) The far-field target amplitude distribution (dashed line), the far-field amplitude distribution given by the GBMs at the DOE plane (solid line), the far-field amplitude distribution from the DOE designed using GBMSO with the Gaussian amplitude input distribution (thick dotted-dashed line), and the far-field amplitude distribution from the DOE designed by the unidirectional method (dotted line). 


$$
\mathrm{PC}=\left(\sum_{i=1}^{N}\left|E\left(k_{i}, 0\right)\right| T_{\text {out }}\left(k_{i}, 0\right) \Delta k\right)^{2},
$$

where $\Delta k$ is the far-field sampling interval. The absolute value of $E\left(k_{i}, 0\right)$ was used, as it was the amplitude distribution of the field that was optimized and the distribution of the phase was left free.

The results of the optimization are shown in Figs. 2 and 3 (with dotted lines) and the figures of merit are given in Table 1 . The $10^{\circ}$ radius rect( ) function far-field distribution was better than the $1^{\circ}$, but the optimized phase that gives the shape of the DOE was much less smooth than for the $10^{\circ}$. Whereas the phase distribution and the figure of merit resulting from the $1^{\circ}$ optimization were independent of the starting phase for all trials, for

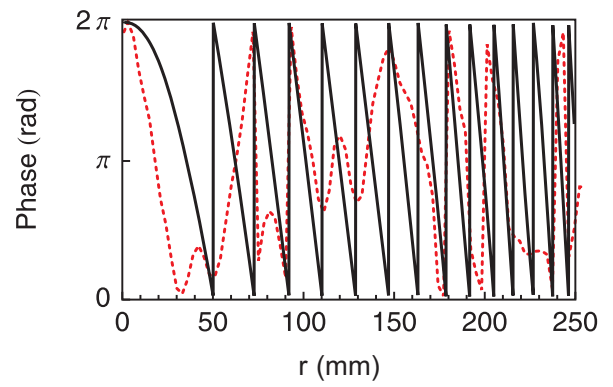

(a)

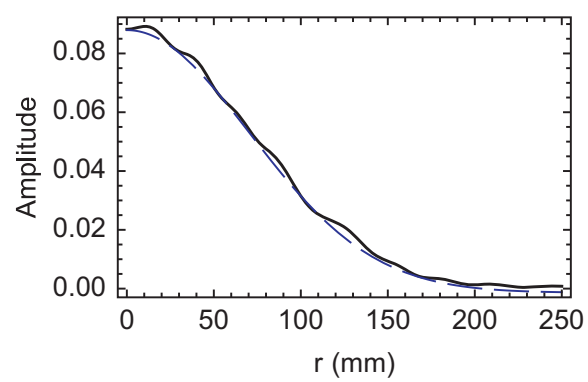

(b)

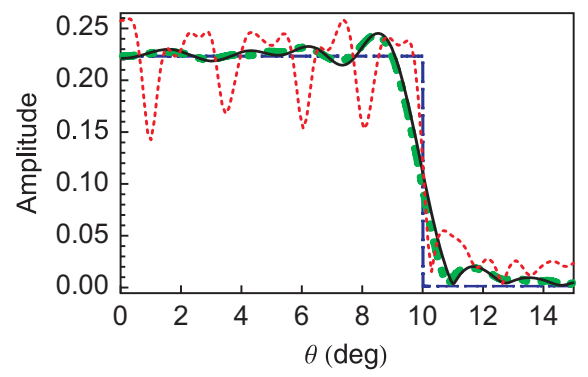

(c)

Fig. 3. (Color online) The results of the optimizations to transform a $100 \mathrm{~mm}$ radius Gaussian input field to a $10^{\circ}$ radius rect( ) function far-field distribution. (a) The phase that gives the DOE profile designed by unidirectional optimization (dotted line) and GBMSO (solid line). (b) The amplitude distribution given by the GBMs at the DOE plane (solid line) and the target amplitude distribution (dashed line). (c) The far-field target amplitude distribution (dashed line), the far-field amplitude distribution given by the GBMs at the DOE plane (solid line), the far-field amplitude distribution from the DOE designed using GBMSO with the Gaussian amplitude input distribution (thick dotted-dashed line), and the far-field amplitude distribution from the DOE designed by the unidirectional method (dotted line).
Table 1. Results of the Optimization to Convert a $\lambda=3 \mathrm{~mm} 100 \mathrm{~mm}$ Gaussian Field to Target $1^{\circ}$ and $10^{\circ}$ Radii rect( ) Function Amplitude Distributions, Using the Unidirectional and GBMSO Methods ${ }^{a}$

\begin{tabular}{ccccccc}
\hline & \multicolumn{2}{c}{ Unidirectional } & & \multicolumn{2}{c}{ GBMSO } \\
\cline { 2 - 3 } \cline { 6 - 7 } rect( ) Function Radius & MSE & PC & & MSE & PC \\
\hline $1^{\circ}$ & 0.001646 & 0.908 & & 0.001474 & 0.917 \\
$10^{\circ}$ & 0.000611 & 0.965 & & 0.000263 & 0.985 \\
\hline
\end{tabular}

${ }^{a}$ For an exact amplitude match, $\mathrm{MSE}=0$ and $\mathrm{PC}=1$.

$10^{\circ}$ they where highly dependent, indicating that a global optimum was not reliably being found.

\section{GBMSO}

\section{A. Introduction}

A beam can be described in terms of GBMs by the following modal expansion $[9,10]$ :

$$
E(x, y, z)=\frac{\sum_{M} \sum_{N} a_{n, m} \exp \left(i \phi_{n, m}\right) \psi_{n, m}\left(w_{0}, x, y, z\right)}{\sum_{M} \sum_{N} a_{n, m}^{2}},
$$

where $a_{n, m}$ and $\phi_{n, m}$ are the amplitude and the phase of the mode coefficients, respectively; $\psi_{n, m}\left(w_{0}, x, y, z\right)$ are the GBMs of waist $w_{0} ; z$ is the propagation distance; and $M$ and $N$ are the maximum index numbers of the mode coefficients used to construct the field. The denominator in Eq. (6) normalizes the power in the field. The GaussHermite basis set, used here, is given by

$$
\begin{aligned}
\psi_{n, m}\left(w_{0}, x, y, z\right)= & \sqrt{\frac{2^{(1 / 2)-n}}{\sqrt{\pi} w n !}} H_{m}\left(\frac{\sqrt{2} x}{w}\right) H_{n}\left(\frac{\sqrt{2} y}{w}\right) \\
& \times \exp \left[-\frac{x^{2}+y^{2}}{w^{2}}-i k z-\frac{i \pi\left(x^{2}+y^{2}\right)}{R(z) \lambda}\right. \\
& \left.+\frac{i \phi_{0}(m+n+1)}{2}\right],
\end{aligned}
$$

where $H_{m}$ and $H_{n}$ are Hermite polynomials of order $m$ and $n$, and the beam radius $w(z)$, the radius of curvature $R(z)$, and the phase slippage $\phi_{0}(z)$, are given by

$$
\begin{aligned}
w(z) & =\sqrt{\left(\frac{z^{2} \lambda^{2}}{\pi^{2} w_{0}^{4}}+1\right) w_{0}^{2}}, \quad R(z)=z\left(\frac{\pi^{2} w_{0}^{4}}{z^{2} \lambda^{2}}+1\right), \\
\phi_{0}(z) & =\tan ^{-1}\left(\frac{z \lambda}{\pi w_{0}^{2}}\right) .
\end{aligned}
$$

The algorithm used to design the DOE-referred to here as GBMSO-involves optimizing the parameters $a_{0,0}, a_{0,1}, \ldots, a_{N, M} ; \phi_{0,0}, \phi_{0,1}, \ldots, \phi_{N, M} ; w_{0}$; and $z$ of Eq. (6) to achieve a field with the prescribed amplitude distributions at the input (DOE) and output (far-field) planes or the closet approximation to it. This was achieved by maximizing the following merit function: 


$$
\begin{aligned}
\eta= & \int_{-\infty}^{\infty} \int_{-\infty}^{\infty}|E(x, y, z)| T_{\text {in }}(x, y) \mathrm{d} x \mathrm{~d} y \\
& +\int_{-\infty}^{\infty} \int_{-\infty}^{\infty}\left|E\left(\theta_{x}, \theta_{y}, z+\bar{z}\right)\right| T_{\text {out }}\left(\theta_{x}, \theta_{y}\right) \mathrm{d} \theta_{x} \mathrm{~d} \theta_{y},
\end{aligned}
$$

where $T_{\text {in }}(x, y)$ is the field that illuminates the DOE, $T_{\text {out }}(x, y)$ is the target amplitude distribution, and $\bar{z}$ is the separation between the input and output planes (if the output plane is located in the far field, $\bar{z}=\infty$ ).

Given a sufficiently large number of modes, any field may be constructed exactly, irrespective of the values of $w_{0}$ and $z$ used; however, a limited number of modes are used in the optimization. Therefore the values $w_{0}$ and $z$ are optimized to more accurately construct the field (in this way the optimal values of the radius of curvature and the radius of the modes were found).

DE was used for the optimization, as the parameter space is nonlinear and DE is a global optimization algorithm. The phase that gives the DOE profile is given by

$$
\phi_{\mathrm{DOE}}(x, y)=\arg \left[E\left(x, y, z_{o}\right)\right],
$$

where $z_{0}$ is the value of $z$ found by the optimization procedure. The field at the DOE is therefore given by

$$
E_{\mathrm{DOE}}(x, y)=T_{i n}(x, y) \exp \left[i \phi_{\mathrm{DOE}}(x, y)\right] .
$$

\section{B. Example: Gaussian Input Field to Uniform Far-Field Distribution}

The following examples were carried out in one dimension for simplicity. In each case the input field $T_{i n}(x, 0)$ was a $\lambda=3 \mathrm{~mm} 100 \mathrm{~mm}$ radius Gaussian with a flat phase-front. The target far-field distributions were rect() functions with far-field angular radii of $1^{\circ}$ and $10^{\circ}$. The modes with even indices between 0 and 20 were used in the optimization (only even indexed modes were used in order to obtain a symmetric DOE).

Whereas DE is capable of finding solutions outside the range of parameter values with which it is initialized, it is more robust if initialized with narrow ranges that encompass the optimum values [5]. In order to construct the DOE plane and output plane target amplitude distributions from the basis modes accurately, the extent of the highest order mode used in the optimization should be at least as large as the extent of the distributions, but not greatly larger. This is the consideration that was applied in choosing the upper and lower limits of $w_{0}$ and $w$ as shown below.

Figure 4 shows the $1^{\circ}$ and $10^{\circ}$ radii target far-field distributions plotted along with the 20th order mode (the highest order mode used in the optimizations); the mode is plotted for the lowest and highest values of $w$ at the DOE plane, and $\theta_{0}$ at the far field. For the $10^{\circ}$ radius rect( ) target function, the lower limits of $\theta_{0}$ and $w$ were

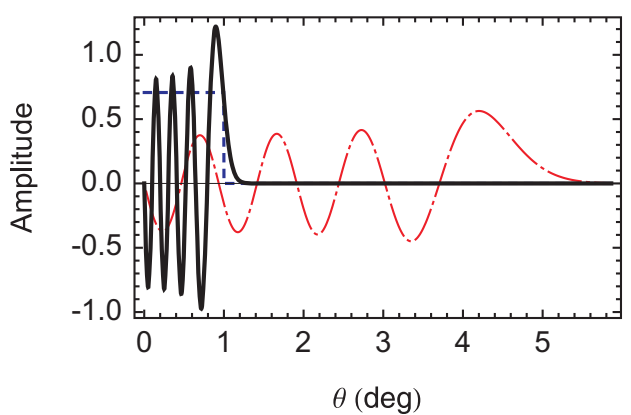

(b)

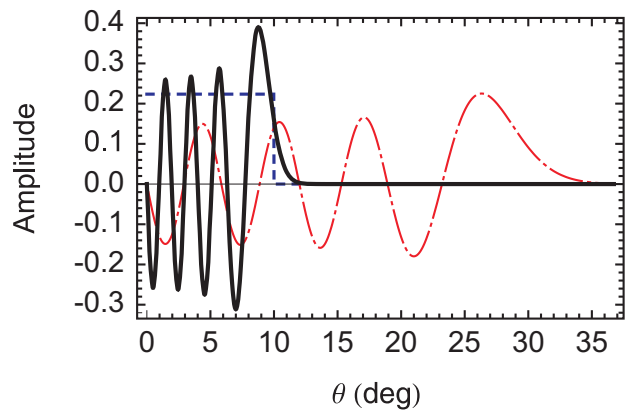

(d)

Fig. 4. (Color online) The upper and lower limits of the random values for the radius of the mode coefficients with which the optimization is initialized were chosen to encompass the likely optimal value (see text). The 20th order mode, the highest order mode used in the optimizations, is shown for a radius at the lower and upper limits (thick solid and dotted-dashed lines, respectively), along with the target amplitude distributions (dashed line). In the far field, the mode radius is given a divergence angle, whereas at the DOE plane, the mode radius is given as a distance. (a) The target (input) Gaussian amplitude distribution for the $1^{\circ}$ target far-field amplitude distribution. (b) The target (output) rect( ) function amplitude distribution for the $1^{\circ}$ target far-field amplitude distribution. (c) The target (input) Gaussian amplitude distribution for the $10^{\circ}$ target far-field amplitude distribution. (d) The target (output) rect( ) function amplitude distribution for the $10^{\circ}$ target far-field amplitude distribution. 
chosen such that the highest order mode used in the optimization had a similar extent to the target distributions at the DOE and far-field planes; the upper limits chosen for $\theta_{0}$ and $w$ were three times the values of the lower limits. The modes were propagated a distance $z$ from the waist location [where the waist radius is $w_{0}=\lambda /\left(\pi \theta_{0}\right)$ ] to a radius of $w$. The propagation distance to the input plane, $z\left(w_{0}, w\right)$, was optimized, rather than optimizing $w$ directly, in order to avoid the generation of infeasible values of $w$ (with $w<w_{0}$ ) during the optimization. $z\left(w_{0}, w\right)$ is given by

$$
z\left(w_{0}, w\right)= \pm \frac{\pi w_{0}^{2}}{\lambda} \sqrt{\frac{w^{2}}{w_{0}^{2}}-1}
$$

The minimum and maximum values of $z$ from the range of values of $w_{0}$ and $w$ were calculated and used to initialize the optimization.

For the $1^{\circ}$ radius target rect( ) function, shown in Figs. 4(a) and 4(b), when $\theta_{0}$ is chosen such that the 20th order mode has a similar extent to the rect( ) function, the minimum mode radius $\left(w=w_{0}\right)$ at the DOE plane is much larger than the target Gaussian distribution, and vice versa when the 20th order mode waist is set to have a similar extent to the Gaussian at the DOE plane; these radii were used as upper and lower limits with which to initialize the optimization, and the optimization found an intermediate mode waist radius.

As the output plane was located in the far field, in Eq. (8), $\bar{z}$ is equal to $\infty$. For the $10^{\circ}$ radius target far field, the optimization was initialized with positive values of $z\left(w_{0}, w\right)$ [it was only necessary to use positive values, as using a negative value of $z\left(w_{0}, w\right)$ along with conjugate values for the mode coefficient in Eq. (6) gave the same amplitude distribution at the target plane] and random values of amplitude and phase of mode coefficients within the ranges $0-1$ and $0-2 \pi$, respectively. The range values of $w_{0}$ and $z$ with which the optimization was initialized are given in Table 2.

The values of $w_{0}$ and $z$ found by the optimizations are given in Table 2 . The results of the optimization for the $1^{\circ}$ and $10^{\circ}$ rect( ) functions are shown in Figs. 2 and 3, respectively. The deviation of the optimized amplitude distribution, $\left|E_{\mathrm{DOE}}(x, y)\right|$, from the target amplitude distribution $T_{i n}(x, y)$ (in this case a Gaussian distribution), shown in Figs. 2(b) and 3(b), causes a corresponding deviation in the far-field distribution when the DOE designed from the optimized phase was used to transform the Gaussian input field. The far field from the DOE with a Gaussian input field is given by

$$
E_{f}\left(k_{x}, k_{y}\right)=\mathcal{F}\left\{T_{i n}(x, y) \exp \left(i \arg \left[E_{\mathrm{DOE}}(x, y)\right]\right)\right\} .
$$

$E_{f}\left(k_{x}, 0\right)$ is shown as a dotted-dashed line in Figs. 2(c) and 3 (c), for the $1^{\circ}$ and $10^{\circ}$ rect ( ) function far-field amplitude distributions, respectively. The PC figures of merit for these fields are given in Table 1 . In both cases this GBMSO method performs better than the unidirectional one.

In the case of the $1^{\circ}$ target rect( ) function distribution, the unidirectional method and GBMSO achieved a comparable performance in terms of the MSE and PC merit functions. In the case of the $10^{\circ}$ target rect( ) function distribution, whereas the MSE of the GBMSO far-field amplitude distribution is reduced by a factor of 2.3 over the unidirectional result, the difference in the PC metric is approximately $2 \%$; thus the relatively small percentage difference in the PC accounts for a large qualitative difference in the shape of the two far-field amplitude distributions.

These algorithms were implemented in Python with the NumPy extension to efficiently compute the FFT. The most computationally expensive part of the optimization for the unidirectional method was the FFT operation of Eq. (3), and for GBMSO it was the computation of the near- and far-field amplitude distributions given by Eq. (6). For GBMSO, the mode set is stored in discretely sampled arrays. Rather than recomputing the entire mode set for each vector, the array containing the discretely sampled modes was calculated only once and the target fields were rescaled to achieve the correct scale relative to the mode set. Each iteration in GBMSO could be computed much more quickly than the unidirectional optimization, as it took approximately 400 times longer to compute the FFT for the unidirectional optimization than to compute the field at both the near- and far-field planes with the dot product for GBMSO.

A significant benefit of GBMSO over the unidirectional approach is that, while producing a better shaped field, the DOE profiles designed are smoother and easier to manufacture. A further advantage of this method is that the output plane need not be at infinity; in fact the beam amplitude distribution can be optimized at one or more intermediate planes as described in the next section.

\section{Multiple Plane Beam Shaping}

The so called "diffraction-free beams," in which the amplitude does not change in form or scale while propagating, were identified in 1987 [11]. These beams have an amplitude cross-section of a Bessel function and are given by

Table 2. Range of $z$ Values after Optimization Was Initialized with Random Values $w$ and $w_{0}$, and Values of

\begin{tabular}{|c|c|c|c|c|c|c|}
\hline \multirow[b]{2}{*}{ rect( ) Function Radius } & \multicolumn{3}{|c|}{$\begin{array}{l}\text { Initialization Range } \\
\qquad(\mathrm{mm})\end{array}$} & \multicolumn{3}{|c|}{$\begin{array}{l}\text { Optimization Result } \\
\qquad(\mathrm{mm})\end{array}$} \\
\hline & $w_{0}$ & $w$ & $z$ & $w_{0}$ & $w$ & $z$ \\
\hline $1^{\circ}$ & $47 \rightarrow 220$ & $47 \rightarrow 220$ & $0 \rightarrow 25,342$ & 90.4 & 107.5 & 5500.8 \\
\hline $10^{\circ}$ & $7.5 \rightarrow 22.5$ & $47 \rightarrow 141$ & $364.4 \rightarrow 3280$ & 11.6 & 55.4 & 661.0 \\
\hline
\end{tabular}
$w_{0}$ and $z$ Found by the Optimization 


$$
E(r, z)=E_{0} \exp \left(i k_{z} z\right) J_{0}\left(k_{r} r\right)
$$

where $J_{0}$ is the zeroth order Bessel function, $k_{z}$ and $k_{r}$ are the longitudinal and radial components of the free-space wave vector related by $k_{0}^{2}=k_{z}^{2}+k_{r}^{2}$, and $k_{0}$ is the free-space wavenumber. Ideal Bessel beams have infinite extent and power-but experimentally generated pseudo-Bessel beams can only approximate them, having finite extent and power. Pseudo-Bessel beams have been generated using dielectric conical shaped lenses called axicons [12-14].

An axicon was designed and fabricated, and the field it produced was measured, by colleagues at the National University of Ireland Maynooth [13]. The high-density polyethylene axicon had a slant angle of $\alpha=20^{\circ}$ and a radius of $30 \mathrm{~mm}$. It was illuminated with a $78 \mathrm{~mm}$ radius Gaussian beam of wavelength $\lambda=3 \mathrm{~mm}$, with a flat phasefront, to achieve a $5 \mathrm{~mm}$ radius central spot over a distance of $150 \mathrm{~mm}$. The simulated field from this axicon is now compared with that of a DOE designed to be "nondiffracting" using GBMSO.

The GBMSO algorithm described above may be extended to control the amplitude distribution of a radially symmetric beam over a region in the near-field of the DOE. Gauss-Laguerre modes are used in place of the
Gauss-Hermite modes used above, as they can efficiently describe circularly symmetric beams. The modeled field is given by

$$
E_{L}(x, y, z)=\frac{\sum_{P} \sum_{M} a_{p, m} \exp \left(i \phi_{p, m}\right) \psi_{p, m}\left(w_{0}, x, y, z\right)}{\sum_{M} \sum_{N} a_{p, m}^{2}},
$$

where $P$ and $M$ are the maximum radial and azimuthal index numbers of the mode coefficients used to construct the field and $\psi_{p m}$ are the Gauss-Laguerre modes given by

$$
\begin{aligned}
& \psi_{p m}=\sqrt{\frac{2}{\pi}} \sqrt{\frac{p !}{(p+|m|) !}} \frac{2^{|m| / 2}\left(\frac{r}{w}\right)^{|m|}}{w} L_{p|m|}\left(\frac{2 \theta^{2}}{w^{2}}\right) \\
& \times \exp \left(i m \phi-\frac{r^{2}}{w^{2}}\right) \text {, }
\end{aligned}
$$

where the radial index $p \geq 0$ and the azimuthal index is $m$. GBMSO is used to design a circularly symmetric DOE that creates a non-diffracting beam over the same range as the beam produced by the axicon described above, by maximizing the following metric:

$$
\begin{aligned}
\bar{\eta}= & 2 \pi \int_{-\infty}^{\infty} \psi_{0,0}\left(w_{\mathrm{spot}}, r, 0\right)\left(\left|E_{L}\left(r, 0,-z_{a}\right)\right|+\left|E_{L}\left(r, 0,+z_{a}\right)\right|\right) r \mathrm{~d} r+W \frac{\int_{z_{1}}^{z_{2}} \psi_{0,0}(w, x=0, z=0)\left|E_{L}(r, 0,+z)\right| \mathrm{d} z}{\sqrt{\int_{z_{1}}^{z_{2}} \psi_{0,0}(w, x=0, z=0)^{2} \mathrm{~d} z} \sqrt{\int_{z_{1}}^{z_{2}}\left|E_{L}(r, 0,+z)\right|^{2} \mathrm{~d} z}} \\
& +2 \pi \int_{-\infty}^{\infty} \psi_{0,0}\left(w_{t}, r, 0\right)\left|E_{L}(r, 0,+z)\right| r \mathrm{~d} r
\end{aligned}
$$

where $\psi_{0,0}\left(w_{\text {spot }}, x, 0\right)$ is the target fundamental Gaussian mode, $\psi_{0,0}\left(w_{t}, r, 0\right)$ is a Gaussian mode of radius $w_{t}$, and $W$ is a weighting constant. As shown in Fig. 5, with this merit function, the optimization searches for a solution

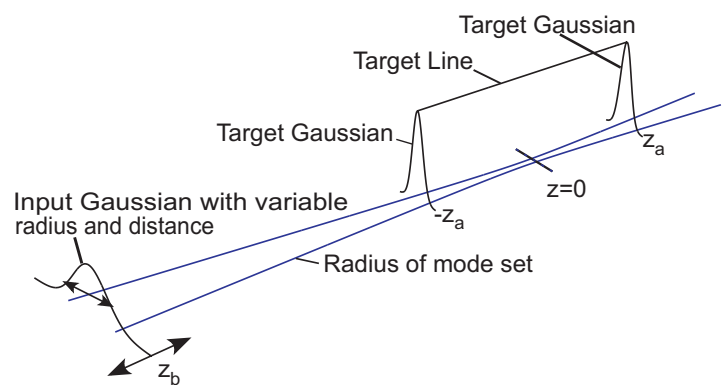

Fig. 5. (Color online) The merit function of Eq. (16) calculates the overlap of the GBM field at the target line and target Gaussian distributions to achieve a non-diffracting beam of constant amplitude at the center of the beam. The propagation distance $z_{b}$ from the DOE plane to the non-diffracting region and the radius of the input Gaussian beam incident on the DOE are optimized. The reference plane, $z=0$, of the GBMs is located equidistant between the two target Gaussians at $\pm z_{a}$. that has a Gaussian amplitude distribution at the extreme planes over which the field was non-diffracting, a uniform central amplitude distribution between these planes, and an input Gaussian for which the radius and distance to the non-diffracting beam were optimized. This merit function was used to achieve a field that is approximately Gaussian, while maintaining a constant amplitude at the center of the beam. Optimizing $w_{t}$ and the propagation distance to the non-diffracting beam allows more freedom to achieve a more optimal solution than fixing these parameters.

In order to reduce the number of parameters to be optimized, the waist radius $w_{0}$ of the modes was fixed during the optimization. In order that the GBMs can describe the target field well, it was critical to minimize the diffraction of the mode set used to construct the field across the non-diffracting region. Therefore, firstly, the waist location was centered between the two target Gaussian beams as shown in Fig. 5 and, secondly, $w_{0}$ was chosen such that $z_{a}$ is the confocal distance, with $w_{0}=\sqrt{z_{a} \lambda / \pi}$.

The optimization was initialized using the first 20 Gauss-Laguerre modes with $w_{\text {spot }}=5 \mathrm{~mm}, z_{a}=75 \mathrm{~mm}$ 


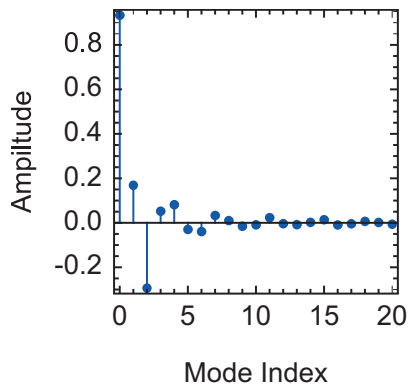

(a)

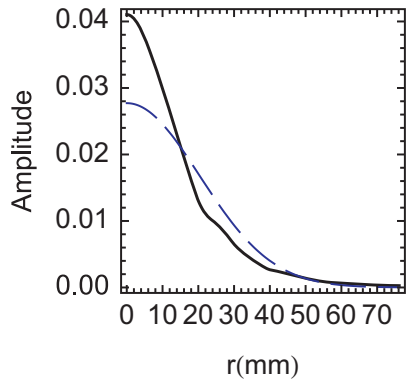

(b)

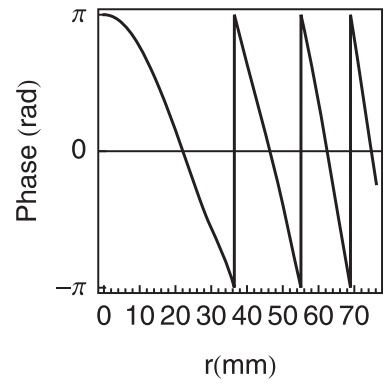

(c)

Fig. 6. (Color online) The results of the optimization to achieve a non-diffracting beam. (a) The amplitude of the mode coefficients found by the optimization. (b) The target amplitude distribution at the DOE plane (dashed line) shown with the amplitude distribution of the optimized solution at this plane (solid line). (c) The phase that gives the shape of the DOE.

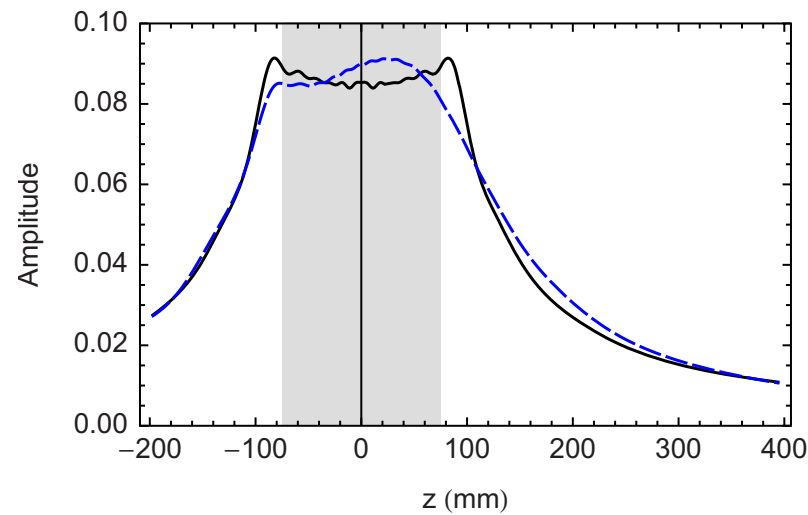

Fig. 7. (Color online) The amplitude at the center of the beam as a function of distance of the non-diffracting beam. The solid line shows the optimized field given by Eq. (14) and the dashed line shows the field from the DOE with the Gaussian input field.

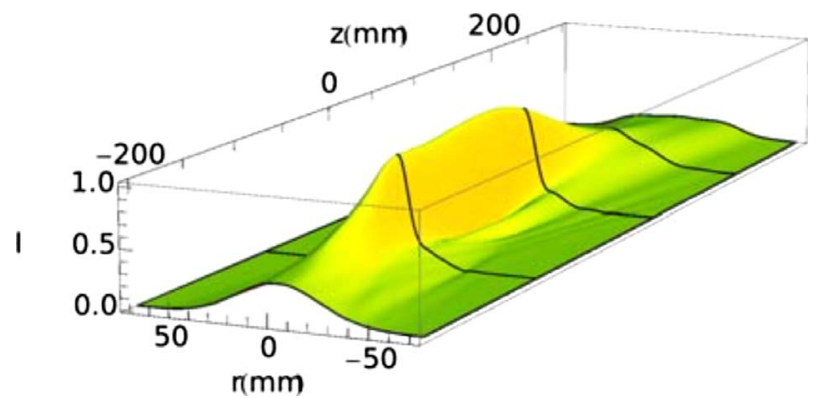

Fig. 8. (Color online) The intensity of the field from the DOE which produces a non-diffracting beam as a function of distance. (giving a total non-diffracting region of $150 \mathrm{~mm}$ ), and $W$ $=10$. Figures 6-9 show the results of the optimization. Figure 6(a) shows the mode coefficients found by the optimization. Figure 6(b) shows a radial cross-section of the amplitude of the field at the DOE plane (at the location found by the optimization, $-197 \mathrm{~mm}$ from the waist location) with the amplitude of the input Gaussian-which has an (optimized) radius of $28.8 \mathrm{~mm}$. A radial crosssection of the phase that gives the shape DOE is shown in Fig. 6(c). The field at the DOE is given by

$$
E_{\mathrm{DOE}}(r, \theta)=\psi_{0,0}\left(w_{t}, r, 0\right) \exp \left(i \arg \left[E\left(r, \theta, z_{b}\right)\right]\right) .
$$

The field at the DOE was reconstructed and propagated using Gauss-Laguerre modes. Figures 7-9 show the propagation of the optimized field $E(r, \theta, z)$ and the simulated field from the DOE from the location of the DOE plane. The optimized field maintains an approximately uniform intensity over the non-diffracting region. The field from the DOE is slightly less uniform due to interference from the field that did not couple to $\psi_{0,0}\left(w_{t}, r, 0\right)$. Figure 9 shows cuts of the simulated field from the DOE in the non-diffracting region and the simulated field from the axicon described earlier. Compared to the beam from the axicon, the field from the GBMSO DOE maintains a greater intensity on-axis for a greater proportion of the non-diffracting region than the field from the axicon.

\section{COMPARISON WITH THE GERCHBERG-SAXTON ALGORITHM}

The widely used Gerchberg-Saxton method was compared to the unidirectional and GBMSO methods elsewhere [7]. For narrow far-field distributions all three methods found similar solutions. As the scale of the target
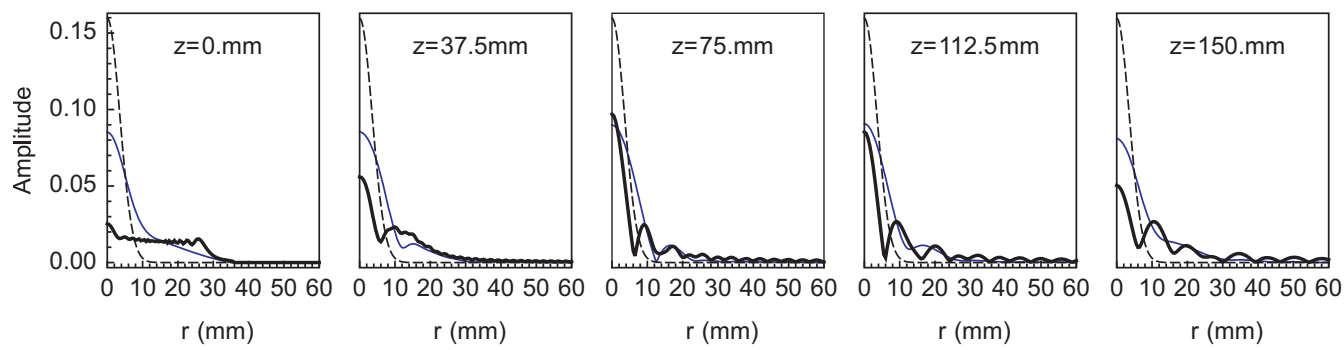

Fig. 9. (Color online) Plots of the amplitude of the field from the axicon (thick solid line) and the field from the DOE designed by optimizing GBMs (thin solid line). These fields are shown with the target $5 \mathrm{~mm}$ radius target Gaussian distribution (dashed line). 
far-field distribution was increased, however, the solution from the Gerchberg-Saxton and unidirectional methods became more dependent on the initial phase distribution; for a $9^{\circ}$ target rect( ) function distribution, after 1000 iterations only $2.3 \%$ of multiple trials achieved the most optimal solution, which was consistently achieved by the GBMSO method. In this paper we have concentrated on comparing the unidirectional method with the GBMSO because, unlike the Gerchberg-Saxton algorithm, they can be used for multiobjective optimization; for example, they can be used to optimize an amplitude distribution over a bandwidth or to optimize the plane of the target distribution.

\section{CONCLUSION}

In this paper, DE was used to design DOEs, using the standard unidirectional method and what we believe to be a new GBMSO approach. Both the phase profile found by the optimization and the linearity of the optimization are dependent on the scale and distribution of the target farfield distribution and the field incident on the DOE. The GBMSO technique was extended to control a beam over a region of the near-field.

For the Gaussian to rect( ) function amplitude distribution conversion, it was found that increasing the scale of the far-field distribution allowed a more optimal solution to be obtained, a relationship which held for a number of other target distributions [7]. GBMSO achieved more optimal solutions than unidirectional and a smoother phase for the $10^{\circ}$ radius rect( ) function far-field target distribution.

GBMSO is more intuitive than the unidirectional method. For a rect( ) function amplitude distribution, the more modes that are used in the reconstruction, the more power can be reconstructed. For beam shaping, the optimal radius of the mode set is determined by the scale of the target distributions at both the input and output planes. Increasing the scale of the target distribution at either the DOE plane or far field allows a greater proportion of the higher order modes to contribute to the power of the optimized field.

GBMSO reduced the number of parameters to be optimized compared with the number required in unidirectional optimization. However, reducing the number of parameters does not necessarily in itself make the problem more or less computationally demanding. With unidirec- tional optimization using the FFT to transform to the far field, trial phase distributions can have arbitrarily high spatial frequencies, and therefore much of the power in the far field may be directed away from the target. This results in the optimization being more likely to get stuck at local optima. GBMSO is much more efficient in this respect, as the powers of the trial near- and far-field distributions are constrained by the extent of the modes, and the DOE design tends to be smoother and therefore easier to manufacture.

\section{REFERENCES}

1. V. Soifer, Methods for Computer Design of Diffractive Optical Elements (Wiley, 2002).

2. H. Kim, B. Yang, and B. Lee, "Iterative Fourier transform algorithm with regularization for the optimal design of diffractive optical elements," J. Opt. Soc. Am. A 21, 2353-2365 (2004).

3. J. Turunen and F. Wyrowski, Diffractive Optics for Industrial and Commercial Applications (Vch Verlagsgesellschaft Mbh, 1998).

4. E. Johnson and M. Abushagur, "Microgenetic-algorithm optimization methods applied to dielectric gratings," J. Opt. Soc. Am. A 12, 1152-1160 (1995)

5. K. Price, R. Storn, and J. Lampinend, Differential Evolution (Springer, 2005).

6. C. Rocha-Alicanoa, D. Covarrubias-Rosalesa, C. BrizuelaRodrigueza, and M. Panduro-Mendozab, "Differential evolution algorithm applied to sidelobe level reduction on a planar array," AEU, Int. J. Electron. Commun. 61, 286-290 (2007).

7. J. Lavelle, "The design and optimisation of quasioptical telescopes," Ph.D. dissertation (NUI Maynooth, 2008).

8. D. O'Shea, T. Suleski, A. Kathman, and D. Prather, Diffractive Optics: Design, Fabrication, and Test (SPIE, 2003).

9. J. Murphy, C. O’Sullivan, W. Lanigan, S. Withington, and N. Trappe, "Modal analysis of the quasi-optical performance of phase gratings," Int. J. Infrared Millim. Waves 20, 1469-1486 (1999).

10. P. Goldsmith, Quasioptical Systems (IEEE, 1997).

11. J. Durnin, "Diffraction free Bessel beams," J. Opt. Soc. Am. A 4, 651-654 (1987).

12. S. Monk, J. Arlt, D. Robertson, J. Courtial, and M. Padgett, "The generation of Bessel beams at millimetre-wave frequencies by use of an axicon," Opt. Commun. 170, 213-215 (1999).

13. N. Trappe, R. Mahon, W. Lanigan, J. A. Murphy, and S. Withington, "The quasi-optical analysis of Bessel beams in the far infrared," Infrared Phys. Technol. 46, 233-247 (2005).

14. R. Di Leonardo, F. Ianni, and G. Ruocco, "Computer generation of optimal holograms for optical trap arrays," Opt. Express 15, 1913-1922 (2007). 